\title{
Leader-Follower Consensus of Second-Order Multiagent Systems with Absent Velocity Measurement and Time Delay
}

\author{
Panpan Yang $\mathbb{D}^{D}$, Ye Tang $\mathbb{D}^{D}$, Maode Yan $\mathbb{D}^{\mathbb{D}}$, and Lei Zuo \\ School of Electronic and Control Engineering, Chang'an University, Xian 710064, China \\ Correspondence should be addressed to Panpan Yang; panpanyang@chd.edu.cn
}

Received 16 July 2018; Accepted 19 September 2018; Published 8 October 2018

Academic Editor: Sebastian Anita

Copyright (C) 2018 Panpan Yang et al. This is an open access article distributed under the Creative Commons Attribution License, which permits unrestricted use, distribution, and reproduction in any medium, provided the original work is properly cited.

\begin{abstract}
The leader-follower consensus problem of second-order multiagent systems with both absent velocity measurement and time delay is considered. First of all, the consensus protocol is designed by introducing an auxiliary system to compensate for the unavailability of the velocity information. Then, time delay is incorporated into the consensus protocol and two cases with, respectively, constant time delay and time-varying delay are investigated. For the case of constant time delay, Lyapunov-Razumikhin theorem is deployed to obtain the sufficient conditions that guarantee the stability of the consensus algorithm. For the case of time-varying delay, the sufficient conditions are also derived by resorting to the Lyapunov-Razumkhin theorem and linear matrix inequalities (LMIs). Various numerical simulations demonstrate the correctness of the theoretical results.
\end{abstract}

\section{Introduction}

Consensus, which aims to make a group of agents reach an agreement on a common value by means of local interactions with each other, has been recognized as a fundamental issue in the cooperative control of multiple mobile autonomous systems $[1,2]$. Over the past decades, the consensus problem of multiagent systems has gained considerable attention from diverse disciplines such as biology, computer science, and control engineering [3-5]. Some achievements have been successfully applied to the distributed sensing of mobile sensor networks, formation keeping of unmanned aerial vehicles, target tracking of swarm robotics, and so forth [6-8].

Among the present literatures on consensus problem, the leader-follower consensus is the most extensively investigated due to its potential applications in many fields, such as the coordinated path tracking of unmanned aerial vehicles (UAVs) [9], the cooperative patrolling of unmanned ground vehicles (UGVs) [10], or the platoon control of intelligent and connected vehicles (ICVs) [11], where followers are required to follow the leader's behavior to fulfill some scheduled tasks. In [12], a consensus based flocking algorithm is proposed with a virtual reference leader. In [13], the leader-follower consensus of Lipschitz nonlinear multiagent systems is investigated under fixed directed communication networks.
For the consensus of leader-follower multiagent systems with uncertainties, an adaptive backstepping sliding mode control algorithm is proposed in [14]. In [15], a distributed extended state observer approach is developed to deal with the unknown external disturbance for the leader-follower consensus of multiagent systems.

However, the existing consensus protocols are basically designed only when the full state of neighboring agents is available; i.e., both the position and the velocity information are required in the consensus of, in particular, second-order multiagent systems. As a matter of fact, the relative velocity measurement between agents is usually more difficult or even impossible to obtain than the relative position measurement in some practical applications [16]. Hence, it is more significant and meaningful to consider the consensus problem without velocity measurement. In [2], the consensus problem for double-integrator multiagent systems without relative velocity measurement is investigated based on a passivity approach. As an extension of [2], the flocking control of multiagent systems without velocity measurement is studied in [17]. In [18], the consensus of heterogeneous multiagent systems without velocity measurement is addressed. In addition, the rotating consensus of multiagent systems in the absence of relative velocity measurement is also studied in [19]. 
On the other hand, communication delay, which is usually called time delay [20], is another important concern in the consensus of multiagent systems because the local interaction relying on the communication networks will inherently induce time delay due to the limited channel bandwidth or communication congestions [21]. By using the root locus method in frequency domain, the second-order consensus problem of multiagent systems with time delay is investigated in [22]. For the leader-follower consensus with time-varying coupling delays, the sufficient conditions are obtained by the algebraic graph theory and LyapunovRazumkhin theorem [23]. In terms of the inequality technique, the consensus of multi-agent systems with nonuniform multiple time-varying delays under both fixed and switching topologies is studied in [24]. In [25], a descriptor model transformation approach is proposed to derive the delay dependent sufficient conditions for the existence of the consensus protocol according to the linear matrix inequalities (LMIs).

Unfortunately, the existing works usually treat the above two issues in separated ways. Literatures that both consider the absent velocity measurement and time delay in the consensus of second-order multiagent systems seem very few. Motivated by this fact, the leader-follower consensus of second-order multiagent systems with both absent velocity measurement and time delay is investigated in this paper. An auxiliary system is firstly designed to compensate for the unavailability of the velocity information and a consensus protocol with absent velocity measurement is proposed. Then, time delay is introduced into the consensus protocol and two cases with, respectively, constant time delay and time-varying delay are discussed. By deploying the Lyapunov-Razumkhin theorem and Lyapunov-Krasovskii theorem, respectively, the sufficient conditions for the stability of the consensus protocol with constant time delay and time-varying delay are derived. Numerical simulations demonstrate the correctness of the theoretical results.

The rest of this paper is organized as follows. In Section 2, some mathematical preliminaries are provided. In Section 3, the leader-follower consensus problem of secondorder multiagent systems with absent velocity measurement and time delay is formulated and a consensus protocol is designed. In Section 4, some theoretical results on the leaderfollower consensus of multiagent systems with absent velocity measurement and time delay are derived. Section 5 gives the numerical simulations to illustrate the correctness of the theoretical results and the concluding remarks are offered in Section 6 .

\section{Mathematical Preliminaries}

In this section, some mathematical backgrounds, including graph theory, matrix theory, and time delay systems are introduced for the theoretical analysis of this paper.

2.1. Graph Theory. In a multiagent system, if each agent is regarded as a node, then its topological structure can be simply described by a graph. Here, graph $\mathscr{G}=(\mathscr{V}, \mathscr{E}, \mathscr{A})$ is a directed graph of order $N$ with a set of nodes $\mathscr{V}=$ $\left\{v_{1}, v_{2}, \ldots, v_{N}\right\}$, a set of edges $\mathscr{E}=\left\{\left(v_{i}, v_{j}\right) \in \mathscr{V} \times \mathscr{V}\right.$ : $\left.v_{i} \sim v_{j}\right\}$, and an adjacency matrix $\mathscr{A}=\left[a_{i j}\right]$, where $a_{i j}$ is the nonnegative adjacency elements of $\mathscr{A}, a_{i j}>0$ if $\left(v_{i}, v_{j}\right) \in \mathscr{E}$ and $a_{i j}=0$, otherwise. The set of neighbors of node $v_{i}$ is denoted by $\mathcal{N}_{i}=\left\{v_{j} \in \mathscr{V}:\left(v_{i}, v_{j}\right) \in \mathscr{E}\right\}$.

If there exists a path from node $i$ to $j$, we say that node $j$ is reachable from $i$. A diagraph $\mathscr{G}$ is strongly connected if any two distinct nodes are reachable from each other. Specifically, graph $\mathscr{G}$ is called an undirected graph if $\left(v_{i}, v_{j}\right) \in$ $\mathscr{E} \Longleftrightarrow\left(v_{j}, v_{i}\right) \in \mathscr{E}$. For an undirected graph, its adjacency matrix is symmetric (i.e., $\mathscr{A}^{\mathrm{T}}=\mathscr{A}$ ) and the corresponding Laplacian matrix $\mathscr{L}$ is defined as $\mathscr{L}=\mathscr{D}-\mathscr{A}$, where $\mathscr{D}=$ $\operatorname{diag}\left\{d_{1}, d_{2}, \ldots, d_{N}\right\} \in \mathbb{R}^{N \times N}$ is the in-degree matrix of graph $\mathscr{G}$ with $d_{i}=\sum_{j=1}^{N} a_{i j}$ being the in-degree of node $v_{i}$. The Laplacian matrix $\mathscr{L}$ is symmetric and positive semidefinite with minimum eigenvalue 0 and the corresponding eigenvector is $\mathbf{1}=[1,1, \ldots, 1]^{\mathrm{T}}$, i.e., $\mathscr{L} \mathbf{1}=0$.

\subsection{Matrix Theory}

Lemma 1. Given vectors $A$ and $B$ with appropriate dimensions, if there exists a symmetric positive definite matrix $Z$ of appropriate dimension, then the following inequality can be obtained:

$$
\pm 2 A^{\mathrm{T}} B \leq A^{\mathrm{T}} Z A+B^{\mathrm{T}} Z^{-1} B
$$

Lemma 2 (Schur complement [26]). If the symmetric matrix $F=F^{\mathrm{T}} \in \mathbb{R}^{(n+m) \times(n+m)}$ can be partitioned as

$$
F=\left[\begin{array}{cc}
A & B^{\mathrm{T}} \\
B & C
\end{array}\right]
$$

where $A \in \mathbb{R}^{n \times n}, B \in \mathbb{R}^{m \times n}$, and $C \in \mathbb{R}^{m \times m}$, then, the following conditions are equivalent:

(1) $F<0$;

(2) $C<0$ and $A-B^{\mathrm{T}} C^{-1} B<0$;

(3) $A<0$ and $C-B A^{-1} B^{\mathrm{T}}<0$.

2.3. Time Delay Systems. Consider the following system:

$$
\begin{aligned}
& \dot{x}(t)=f\left(t, x_{t}\right), \quad t>0 \\
& x(\theta)=\varphi(\theta), \quad \theta \in[-\tau, 0]
\end{aligned}
$$

where $x_{t}(\theta)=x(t+\theta)$ for $\forall \theta \in[-\tau, 0]$ and $f(t, 0)=0$. Let $\mathbb{C}\left([-\tau, 0], \mathbb{R}^{n}\right)$ be a Banach space of continuous functions defined on an interval $[-\tau, 0]$, taking values in $\mathbb{R}^{n}$ with the topology of uniform convergence, and with a norm $\|\varphi\|_{c}=$ $\max _{\theta \in[-\tau, 0]}\|\varphi(\theta)\|$. Then, the following results for the stability of system (3) can be obtained.

Lemma 3 (Lyapunov-Razumikhin theorem [27]). Let $\phi_{1}(s)$, $\phi_{2}(s)$, and $\phi_{3}(s)$ be continuous, nonnegative, and nondecreasing functions with $\phi_{1}(s)>0, \phi_{2}(s)>0, \phi_{3}(s)>0$ for $s>0$ and $\phi_{1}(0)=\phi_{2}(0)=0$. For system (3), the function $f: \mathbb{C}\left([-\tau, 0], \mathbb{R}^{n}\right) \longrightarrow R$ is supposed to take bounded sets of $\mathbb{C}\left([-\tau, 0], \mathbb{R}^{n}\right)$ in bounded sets of $\mathbb{R}^{n}$. 
(1) If there exists a continuous function $V(t, x)$ with $t \in R$ and $x \in \mathbb{R}^{n}$ such that

$$
\phi_{1}(\|x\|) \leq V(t, x) \leq \phi_{2}(\|x\|)
$$

(2) if there exists a continuous nondecreasing function $\phi(s)$ with $\phi(s)>0, s>0$ such that

$$
\begin{aligned}
\dot{V}(t, x) & \leq-\phi_{3}(\|x\|) \\
\text { if } V(t+\theta, x(t+\theta)) & <\phi(V(t, x(t))), \quad \theta \in[-\tau, 0]
\end{aligned}
$$

then the solution $x=0$ is uniformly asymptotically stable.

Usually, $V(t, x)$ is called Lyapunov-Razumikhin function if it satisfies both (4) and (5) in Lemma 3.

Remark 4. It can be seen in Lemma 3 that one only needs to consider the initial data if a trajectory of (3) starting from these initial data is "diverging" rather than to require that $\dot{V}(t, x)$ be nonpositive for all initial data in order to have the stability of system (3). delay:

Consider the following differential equation with time

$$
\dot{x}(t)=f\left(t, x_{t}\right), \quad t \geq t_{0}
$$

where $x(t) \in \mathbb{R}^{n}$ is a state vector. In addition, $x_{t}(\theta)$ denotes a transfer operator of state trajectory for $[-\tau, 0]$ and is defined as $x_{t}(\theta)=x(t+\theta)$ for $\forall \theta \in[-\tau, 0]$. Functional $f\left(t, x_{t}\right)$ is continuous for $x_{t}$ and satisfies $f(t, 0)=0$.

Lemma 5 (Lyapunov-Krasovskii theorem [27]). Supposing that the mapping $f: \mathbb{R} \times \mathbb{C} \longrightarrow \mathbb{R}^{n}$ is continuous and nondecreasing $\left(u, v, w: \overline{\mathbb{R}}_{+} \longrightarrow \overline{\mathbb{R}}_{+}\right.$. For $s>0, u(s)>0$, $v(s)>0$; for $s=0, u(s)=v(s)=0$ ), the stability of system (6) can be proved if it satisfies the following:

(1) $V: \mathbb{R} \times \mathbb{C} \longrightarrow \mathbb{R}$ is continuous and differentiable;

(2) $u\|\phi(0)\| \leq V(t, \phi) \leq v\|\phi\|_{\mathcal{C}}$;

(3) $\dot{V}(t, \phi) \leq-\omega(\|\phi(0)\|)$.

In addition, the solution $x=0$ is uniformly asymptotically stable for $s>0, \omega(s)>0$, and globally uniformly asymptotically stable for $\lim _{s \rightarrow \infty} u(s)=\infty$.

\section{Problem Formulation}

3.1. Problem Statement. Considering a multiagent system with one leader and $N$ followers, the dynamics of the followers are governed by

$$
\begin{aligned}
& \dot{x}_{i}=v_{i} \\
& \dot{v}_{i}=u_{i}
\end{aligned}
$$

where $x_{i}, v_{i} \in \mathbb{R}^{n}$ are the position and velocity of agent $i$ and $u_{i}$ is the acceleration vector and is taken as the control input.

The dynamics of the leader is expressed as

$$
\dot{x}_{L}=v_{L}
$$

where $v_{L}$ is the desired constant velocity.
For multiagent system (7), each agent is supposed to be mounted with GPS equipment to get its position information and wireless communication device to transmit its position to other agents. Then, the following definition is given.

Definition 6. The leader-follower consensus of second-order multiagent systems is solved, if for any initial conditions, the following hold:

$$
\begin{aligned}
& \lim _{t \rightarrow \infty}\left|x_{i}-x_{L}\right|=0 \\
& \lim _{t \rightarrow \infty}\left|v_{i}-v_{L}\right|=0
\end{aligned}
$$

Form (9) it can be seen that the consensus is reached if the position and velocity of followers both converge to that of the leader. However, the velocity information is unmeasurable in some practical occasions and one needs to fulfill the consensus of multiagent systems based on position information only. In addition, the position information transmission between agents may be delayed due to the limited channel bandwidth or communication congestions.

Therefore, the main objective of this paper is to synthesis the distributed consensus protocol for second-order multiagent systems such that when the velocity information is unavailable and there exist time delays during the position information transmission between agents, the consensus of all agents will asymptotically be achieved.

3.2. Consensus Protocol Design. For the consensus of secondorder multiagent systems without velocity measurement, the distributed consensus protocol can be formulated as

$$
\begin{aligned}
u_{i}= & -\sum_{j=1}^{n} a_{i j}\left(x_{i}(t)-x_{j}(t)\right)-b_{i}\left(x_{i}(t)-x_{L}(t)\right) \\
& +k_{1} \dot{y}_{i}+\dot{v}_{L}
\end{aligned}
$$

where $k_{1}>0$ is the control gain and $b_{i} \in[0,1]$ represents whether agent $i$ directly communicates with the leader; i.e., if agent $i$ can obtain the position information of the leader, we say $b_{i}=1$; otherwise, $b_{i}=0$. In addition, $y_{i} \in \mathbb{R}^{n}$ is a specially designed auxiliary system compensating for the unavailable velocity information, which is expressed as

$$
\begin{gathered}
\dot{y}_{i}=-k_{2} y_{i}-k_{3} b_{i}\left(x_{i}(t)-x_{L}(t)\right) \\
-k_{3} \sum_{j=1}^{n} a_{i j}\left(x_{i}(t)-x_{j}(t)\right)
\end{gathered}
$$

where $k_{2}, k_{3}>0$ are the control gains.

If time delay is considered in the position information transmission between agents, the consensus protocol can then be written as

$$
\begin{aligned}
u_{i}= & -\sum_{j=1}^{n} a_{i j}\left(x_{i}(t-\tau(t))-x_{j}(t-\tau(t))\right) \\
& -b_{i}\left(x_{i}(t-\tau(t))-x_{L}(t-\tau(t))\right)+k_{1} \dot{y}_{i}+\dot{v}_{L}
\end{aligned}
$$




$$
\begin{array}{r}
\dot{y}_{i}=-k_{2} y_{i}-k_{3} b_{i}\left(x_{i}(t-\tau(t))-x_{L}(t-\tau(t))\right) \\
-k_{3} \sum_{j=1}^{n} a_{i j}\left(x_{i}(t-\tau(t))-x_{j}(t-\tau(t))\right)
\end{array}
$$

where $\tau(t)$ denotes the time delay.

Let $\tilde{x}_{i}(t-\tau(t))=x_{i}(t-\tau(t))-x_{L}(t-\tau(t))$ and $\widetilde{v}_{i}(t-\tau(t))=$ $v_{i}(t-\tau(t))-v_{L}$ be the position and velocity errors of agent $i$ with respect to the leader. Then, (12) is rewritten as

$$
\begin{aligned}
u_{i}= & -\sum_{j=1}^{n} a_{i j}\left(\tilde{x}_{i}(t-\tau(t))-\tilde{x}_{j}(t-\tau(t))\right) \\
& -b_{i} \tilde{x}_{i}(t-\tau(t))+k_{1} \dot{y}_{i}+\dot{v}_{L} \\
\dot{y}_{i}= & -k_{2} y_{i}-k_{3} b_{i} \tilde{x}_{i}(t-\tau(t)) \\
& -k_{3} \sum_{j=1}^{n} a_{i j}\left(\tilde{x}_{i}(t-\tau(t))-\tilde{x}_{j}(t-\tau(t))\right)
\end{aligned}
$$

For simplicity, we denote

$$
\begin{gathered}
\tilde{x}=\left[\begin{array}{c}
\widetilde{x}_{1} \\
\widetilde{x}_{2} \\
\vdots \\
\tilde{x}_{n}
\end{array}\right], \\
\widetilde{v}=\left[\begin{array}{c}
\widetilde{v}_{1} \\
\widetilde{v}_{2} \\
\vdots \\
\widetilde{v}_{n}
\end{array}\right], \\
y=\left[\begin{array}{c}
y_{1} \\
y_{2} \\
\vdots \\
y_{n}
\end{array}\right]
\end{gathered}
$$

Then, the error dynamics of multiagent system (7) can be presented as

$$
\begin{aligned}
& \dot{\tilde{x}}=\widetilde{v} \\
& \dot{\tilde{v}}=-(\mathscr{L}+B) \tilde{x}(t-\tau(t))+k_{1} \dot{y} \\
& \dot{y}=-k_{2} y-k_{3}(\mathscr{L}+B) \tilde{x}(t-\tau(t))
\end{aligned}
$$

where $B=\operatorname{diag}\left\{b_{1}, b_{2} \ldots b_{n}\right\}$ is a $n \times n$ matrix and $\mathscr{L}$ is the Laplacian matrix.

Remark 7. From (15) we can see that, by introducing an auxiliary system $y_{i}$ into the consensus protocol (10), the original second-order multiagent system (7) is transformed into a third-order-alike multiagent system. In the following, we will give a detailed theoretical analysis on the stability of consensus protocol (15).

\section{Main Results}

In this section, two time delay cases, including constant time delay and time-varying delay, are considered in the consensus of second-order multiagent systems with absent velocity measurement. Some theoretical results are derived for the stability of the proposed consensus algorithm (15) in the presence of time delay.

4.1. Consensus of Second-Order Multiagent Systems with Absent Velocity Measurement and Constant Time Delay. For the case of constant time delay, $\tau(t)$ is assumed to be a constant value, i.e., $\tau(t)=\tau$. Then, (15) can be written in the following state space form

$$
\begin{aligned}
{\left[\begin{array}{c}
\dot{\tilde{x}} \\
\dot{\tilde{v}} \\
\dot{y}
\end{array}\right]=} & {\left[\begin{array}{ccc}
\mathbf{0} & I_{n} & \mathbf{0} \\
\mathbf{0} & \mathbf{0} & -k_{1} k_{2} I_{n} \\
\mathbf{0} & \mathbf{0} & -k_{2} I_{n}
\end{array}\right]\left[\begin{array}{l}
\tilde{x} \\
\tilde{v} \\
y
\end{array}\right] } \\
& +\left[\begin{array}{ccc}
-\left(1+k_{1} k_{3}\right) H & \mathbf{0} & \mathbf{0} \\
-k_{3} H & \mathbf{0} & \mathbf{0}
\end{array}\right]\left[\begin{array}{l}
\tilde{x}(t-\tau) \\
\tilde{v}(t-\tau) \\
y(t-\tau)
\end{array}\right]
\end{aligned}
$$

where $H=\mathscr{L}+B$.

Let $\xi^{\mathrm{T}}=\left[\begin{array}{lll}\tilde{x}^{\mathrm{T}} & \tilde{v}^{\mathrm{T}} & y^{\mathrm{T}}\end{array}\right]$; we have

$$
\dot{\xi}(t)=C_{0} \xi(t)+C_{1} \xi(t-\tau)
$$

where

$$
\begin{aligned}
C_{0} & =\left[\begin{array}{ccc}
\mathbf{0} & I_{n} & \mathbf{0} \\
\mathbf{0} & \mathbf{0} & -k_{1} k_{2} I_{n} \\
\mathbf{0} & \mathbf{0} & -k_{2} I_{n}
\end{array}\right], \\
C_{1} & =\left[\begin{array}{cccc}
\mathbf{0} & \mathbf{0} & \mathbf{0} \\
-\left(1+k_{1} k_{3}\right) H & \mathbf{0} & \mathbf{0} \\
-k_{3} H & \mathbf{0} & \mathbf{0}
\end{array}\right]
\end{aligned}
$$

Then, the following theorem for the stability of secondorder multiagent systems with absent velocity measurement and constant time delay is obtained.

Theorem 8. Consider the multiagent system (16) with constant time delay $\tau$, if $\tau$ satisfies

$$
\tau<\tau^{*}=\frac{\lambda_{\min }(F)}{\left\|Q_{1}\right\|+\left\|Q_{2}\right\|+2 r\|P\|}
$$

where $F=-\left[P\left(C_{0}+C_{1}\right)+\left(C_{0}+C_{1}\right)^{\mathrm{T}} P\right]$ is a positive definite symmetric matrix and $Q_{1}$ and $Q_{2}$ are defined by $Q_{1}=$ $P C_{1} C_{0} P^{-1} C_{0}^{\mathrm{T}} C_{1}^{\mathrm{T}} P$ and $Q_{2}=P\left(C_{1}^{2}\right) P^{-1}\left(C_{1}^{2}\right)^{\mathrm{T}} P$, respectively.

Then, the consensus of multiagent system (16) is asymptotically achieved. 
Proof. Choose the following Lyapunov-Razumikhin function:

$$
V(\xi)=\xi^{\mathrm{T}} P \xi
$$

where $P$ is a positive definite matrix.

Taking the derivative of $V(\xi)$ yields

$$
\dot{V}(\xi)=2 \xi^{\mathrm{T}} P \dot{\xi}
$$

According to the Leibniz-Newton formula and (17), the following holds:

$$
\begin{aligned}
\xi(t) & -\xi(t-\tau)=\int_{t-\tau}^{t} \dot{\xi}(s) d s \\
= & \int_{-\tau}^{0}\left[C_{0} \xi(t+s)+C_{1} \xi(t+s-\tau)\right] d s
\end{aligned}
$$

Thus

$$
\begin{aligned}
\xi(t-\tau)= & \xi(t)-\int_{t-\tau}^{t} \dot{\xi}(s) d s \\
= & \xi(t)-C_{0} \int_{-\tau}^{0} \xi(t+s) d s \\
& -C_{1} \int_{-2 \tau}^{-\tau} \xi(t+s) d s
\end{aligned}
$$

Then, (17) can be rewritten as

$$
\begin{aligned}
\dot{\xi}(t)= & C_{0} \xi(t)+C_{1}\left[\xi(t)-\int_{t-\tau}^{t} \dot{\xi}(s) d s\right] \\
= & \left(C_{0}+C_{1}\right) \xi(t)-C_{1} C_{0} \int_{-\tau}^{0} \xi(t+s) d s \\
& -C_{1}^{2} \int_{-2 h}^{-\tau} \xi(t+s) d s
\end{aligned}
$$

Invoking (24), the time derivative of $V(\xi)$ is that

$$
\begin{aligned}
\dot{V}(\xi)= & 2 \xi^{\mathrm{T}} P\left(C_{0}+C_{1}\right) \xi-2 \xi^{\mathrm{T}} P C_{1} C_{0} \int_{-\tau}^{0} \xi(t+s) d s \\
& -2 \xi^{\mathrm{T}} P C_{1}^{2} \int_{-2 \tau}^{-\tau} \xi(t+s) d s
\end{aligned}
$$

In addition, the following inequalities hold according to Lemma 1:

$$
\begin{gathered}
-2 \xi^{\mathrm{T}} P C_{1} C_{0} \int_{-\tau}^{0} \xi(t+s) d s \\
\leq \tau \xi^{\mathrm{T}} P C_{1} C_{0} P^{-1} C_{0}^{\mathrm{T}} C_{1}^{\mathrm{T}} P \\
+\int_{-\tau}^{0} \xi(t+s)^{\mathrm{T}} P \xi(t+s) d s \\
-2 \xi^{\mathrm{T}} P C_{1}^{2} \int_{-2 \tau}^{-\tau} \xi(t+s) d s \\
\leq \tau \xi^{\mathrm{T}} P C_{1}^{2} P^{-1}\left(C_{1}^{2}\right)^{\mathrm{T}} P \xi \\
+\int_{-2 \tau}^{-\tau} \xi(t+s)^{\mathrm{T}} P \xi(t+s) d s
\end{gathered}
$$

Therefore, (25) can be expressed as

$$
\begin{aligned}
\dot{V}(\xi)= & -\xi^{\mathrm{T}} F \xi-2 \xi^{\mathrm{T}} P C_{1} C_{0} \int_{-\tau}^{0} \xi(t+s) d s \\
& -2 \xi^{\mathrm{T}} P C_{1}^{2} \int_{-2 \tau}^{-\tau} \xi(t+s) d s \\
\leq & -\xi^{\mathrm{T}} F \xi+\tau \xi^{\mathrm{T}} P C_{1} C_{0} P^{-1} C_{0}^{\mathrm{T}} C_{1}^{\mathrm{T}} P \xi \\
& +\int_{-\tau}^{0} \xi(t+s)^{\mathrm{T}} P \xi(t+s) d s \\
& +\tau \xi^{\mathrm{T}} P C_{1}^{2} P^{-1}\left(C_{1}^{2}\right)^{\mathrm{T}} P \xi \\
& +\int_{-2 \tau}^{-\tau} \xi(t+s)^{\mathrm{T}} P \xi(t+s) d s \\
\leq & -\xi^{\mathrm{T}} F \xi+\tau \xi^{\mathrm{T}} P C_{1} C_{0} P^{-1} C_{0}^{\mathrm{T}} C_{1}^{\mathrm{T}} P \xi \\
& +\tau \xi^{\mathrm{T}} P C_{1}^{2} P^{-1}\left(C_{1}^{2}\right)^{\mathrm{T}} P \xi \\
& +\int_{-2 \tau}^{0} \xi(t+s)^{\mathrm{T}} P \xi(t+s) d s
\end{aligned}
$$

Let $\phi(s)=r s$ and $r>1$. According to Lemma 3, we have

$$
\xi(t+s)^{\mathrm{T}} P \xi(t+s) \leq r \xi^{\mathrm{T}} P \xi
$$

when

$$
V(\xi(t+\omega))<r V(\xi(\omega)), \quad-\tau \leq \omega \leq 0
$$

Note that (29) satisfies the following inequality according to mean value theorems for definite integrals:

$$
\int_{-2 \tau}^{0} \xi(t+s)^{\mathrm{T}} P \xi(t+s) d s \leq 2 r \tau \xi^{\mathrm{T}} P \xi
$$

Finally, invoking (28) and (31), $\dot{V}(\xi)$ follows that

$$
\dot{V}(\xi) \leq-\xi^{\mathrm{T}}\left[F-\tau\left(Q_{1}+Q_{2}+2 r P\right)\right] \xi<0
$$

Thus, we know

$$
\lim _{t \rightarrow \infty} \xi(t)=0
$$

that is, $\tilde{x}(t-\tau)=x_{i}(t-\tau)-x_{L}(t-\tau) \longrightarrow 0, \widetilde{v}(t-\tau)=$ $v_{i}(t-\tau)-v_{L} \longrightarrow 0$, which means that the position and velocity of followers will asymptotically converge to that of the leader and thus the consensus is achieved.

Remark 9. Note that the above assumption that the time delay in the position information transmission between agents is a constant value may not be reasonable in some practical applications, as the time delay caused by unreliable communication networks may be time-varying and even a stochastic value [28]. Therefore, the consensus of secondorder multiagent systems with absent velocity measurement and time-varying delay will be investigated in the following contents. 
4.2. Consensus of Second-Order Multiagent Systems with Absent Velocity Measurement and Time-Varying Delay. For the case of time-varying delay, $\tau(t)$ varies over time. Assume that $\tau(t)$ is a continuously differentiable function satisfying $0 \leq \tau(t) \leq \tau^{*}$ and $\dot{\tau}(t) \leq h<1$, for all $t \geq 0$. Similarly, (15) can be written in the following state space form:

$$
\begin{aligned}
{\left[\begin{array}{c}
\dot{\tilde{x}} \\
\dot{\tilde{v}} \\
\dot{y}
\end{array}\right]=} & {\left[\begin{array}{ccc}
\mathbf{0} & I_{n} & \mathbf{0} \\
\mathbf{0} & \mathbf{0} & -k_{1} k_{2} I_{n} \\
\mathbf{0} & \mathbf{0} & -k_{2} I_{n}
\end{array}\right]\left[\begin{array}{l}
\tilde{x} \\
\tilde{v} \\
y
\end{array}\right] } \\
& +\left[\begin{array}{ccc}
-\left(1+k_{1} k_{3}\right) H & \mathbf{0} & \mathbf{0} \\
-k_{3} H & \mathbf{0} & \mathbf{0}
\end{array}\right]\left[\begin{array}{l}
\tilde{x}(t-\tau(t)) \\
\tilde{v}(t-\tau(t)) \\
y(t-\tau(t))
\end{array}\right]
\end{aligned}
$$

where $H=\mathscr{L}+B$.

Let $\xi^{\mathrm{T}}=\left[\begin{array}{lll}\tilde{x}^{\mathrm{T}} & \tilde{v}^{\mathrm{T}} & y^{\mathrm{T}}\end{array}\right]$; we have

$$
\dot{\xi}(t)=C_{0} \xi(t)+C_{1} \xi(t-\tau(t))
$$

where

$$
\begin{aligned}
C_{0} & =\left[\begin{array}{ccc}
\mathbf{0} & I_{n} & \mathbf{0} \\
\mathbf{0} & \mathbf{0} & -k_{1} k_{2} I_{n} \\
\mathbf{0} & \mathbf{0} & -k_{2} I_{n}
\end{array}\right], \\
C_{1} & =\left[\begin{array}{cccc}
\mathbf{0} & \mathbf{0} & \mathbf{0} \\
-\left(1+k_{1} k_{3}\right) H & \mathbf{0} & \mathbf{0} \\
-k_{3} H & \mathbf{0} & \mathbf{0}
\end{array}\right]
\end{aligned}
$$

Then, the following theorem for the stability of secondorder multiagent systems with absent velocity measurement and time-varying delay is introduced.

Theorem 10. Consider the multiagent system (34) with timevarying delay $\tau(t)$, if there exist symmetric positive definite matrices $G, R$, and $E$ of appropriate dimensions satisfying the following inequality:

$$
\Lambda=\left[\begin{array}{cccc}
\Lambda_{11} & \Lambda_{12} & G & \tau^{*} G C_{1} \\
\Lambda_{21} & \Lambda_{22} & 0 & 0 \\
G & 0 & -R & 0 \\
\tau^{*} G C_{1} & 0 & 0 & -\tau^{*} R
\end{array}\right]<0
$$

where $\Lambda_{11}=\left(C_{0}+C_{1}\right)^{\mathrm{T}} G+G\left(C_{0}+C_{1}\right)+E+\tau^{*}\left(C_{0}+C_{1}\right)^{\mathrm{T}} R\left(C_{0}+\right.$ $\left.C_{1}\right)-(1-h) E, \Lambda_{12}=(1-h) E-2 \tau^{*}\left(C_{0}+C_{1}\right)^{\mathrm{T}} R C_{1}, \Lambda_{21}=$ $(1-h) E$, and $\Lambda_{22}=\tau^{*} C_{1}{ }^{\mathrm{T}} R C_{1}-(1-h) E$.

Then, the consensus of second-order multiagent system (34) with time-varying delay is asymptotically achieved.

Proof. Define the following Lyapunov-Krasovskii functional:

$$
\begin{aligned}
V_{2}(t)= & \xi^{\mathrm{T}}(t) G \xi(t)+\int_{t-\tau(t)}^{t} \xi^{\mathrm{T}}(s) E \xi(s) d s \\
& +\int_{-\tau(t)}^{0} \int_{t+\theta}^{t} \xi^{\mathrm{T}}(s) R \xi(s) d s d \theta
\end{aligned}
$$

Taking the time derivative of $V_{2}(t)$ yields

$$
\begin{aligned}
& \dot{V}_{2}(t)=\dot{\xi}^{\mathrm{T}}(t) G \xi(t)+\xi^{\mathrm{T}}(t) G \dot{\xi}(t)+\xi^{\mathrm{T}}(t) E \xi(t) \\
& -(1-\dot{\tau}(t)) \xi^{\mathrm{T}}(t-\tau(t)) E \xi(t-\tau(t))+\tau(t) \dot{\xi}^{\mathrm{T}}(t) \\
& \cdot R \dot{\xi}(t)-\int_{t-\tau(t)}^{t} \dot{\xi}^{\mathrm{T}}(s) R \dot{\xi}(s) d s=\left[\left(C_{0}+C_{1}\right) \xi(t)\right. \\
& \left.-C_{1} \int_{t-\tau(t)}^{t} \dot{\xi}(s) d s\right]^{\mathrm{T}} G \xi(t)+\xi^{\mathrm{T}}(t) \\
& \cdot G\left[\left(C_{0}+C_{1}\right) \xi(t)-C_{1} \int_{t-\tau(t)}^{t} \dot{\xi}(s) d s\right]+\xi^{\mathrm{T}}(t) \\
& E \xi(t)+\tau(t) \dot{\xi}^{\mathrm{T}}(t) R \dot{\xi}(t)-\int_{t-\tau(t)}^{t} \dot{\xi}^{\mathrm{T}}(s) R \dot{\xi}(s) d s \\
& -(1-\dot{\tau}(t)) \xi^{\mathrm{T}}(t-\tau(t)) E \xi(t-\tau(t))=\xi^{\mathrm{T}}(t) \\
& \cdot\left[\left(C_{0}+C_{1}\right)^{\mathrm{T}} G+G\left(C_{0}+C_{1}\right)+E\right] \xi(t) \\
& -\int_{t-\tau(t)}^{t} \dot{\xi}^{\mathrm{T}}(s) C_{1}^{\mathrm{T}} G \xi(t) d s \\
& -\int_{t-\tau(t)}^{t} \xi^{\mathrm{T}}(t) G C_{1} \dot{\xi}(s) d s-(1-\dot{\tau}(t)) \xi^{\mathrm{T}}(t \\
& -\tau(t)) E \xi(t-\tau(t))-\int_{t-\tau(t)}^{t} \dot{\xi}^{\mathrm{T}}(s) R \dot{\xi}(s) d s \\
& +\tau(t) \dot{\xi}^{\mathrm{T}}(t) R \dot{\xi}(t) \leq \xi^{\mathrm{T}}(t)\left[\left(C_{0}+C_{1}\right)^{\mathrm{T}} G\right. \\
& \left.+G\left(C_{0}+C_{1}\right)+E\right] \xi(t)+\frac{1}{2} \int_{t-\tau(t)}^{t} \dot{\xi}^{\mathrm{T}}(s) R \dot{\xi}(s) d s \\
& +\frac{1}{2} \int_{t-\tau(t)}^{t} \xi^{\mathrm{T}}(t) G C_{1} R^{-1} C_{1}^{\mathrm{T}} G \xi(t) d s+\frac{1}{2} \\
& \cdot \int_{t-\tau(t)}^{t} \dot{\xi}^{\mathrm{T}}(s) R \dot{\xi}(s) d s+\frac{1}{2} \\
& \cdot \int_{t-\tau(t)}^{t} \xi^{\mathrm{T}}(t) G C_{1} R^{-1} C_{1}^{\mathrm{T}} G \xi(t) d s \\
& -\int_{t-\tau(t)}^{t} \dot{\xi}^{\mathrm{T}}(s) R \dot{\xi}(s) d s-(1-h) \xi^{\mathrm{T}}(t-\tau(t)) \\
& E \xi(t-\tau(t))+\tau(t) \dot{\xi}^{\mathrm{T}}(t) R \dot{\xi}(t) \leq \xi^{\mathrm{T}}(t) \\
& \cdot\left[\left(C_{0}+C_{1}\right)^{\mathrm{T}} G+G\left(C_{0}+C_{1}\right)+E\right. \\
& \left.+\tau(t) G C_{1} R^{-1} C_{1}^{\mathrm{T}} G+G R^{-1} G\right] \xi(t)-(1-h) \xi^{\mathrm{T}}(t \\
& -\tau(t)) E \xi(t-\tau(t))+\tau(t) \dot{\xi}^{\mathrm{T}}(t) R \dot{\xi}(t)=\xi^{\mathrm{T}}(t) \\
& \cdot\left[\left(C_{0}+C_{1}\right)^{\mathrm{T}} G+G\left(C_{0}+C_{1}\right)+E\right. \\
& \left.+\tau(t) G C_{1} R^{-1} C_{1}^{\mathrm{T}} G+G R^{-1} G\right] \xi(t)-(1-h) \xi^{\mathrm{T}}(t
\end{aligned}
$$




$$
\begin{aligned}
& -\tau(t)) E \xi(t-\tau(t))+\tau(t)\left[\left(C_{0}+C_{1}\right) \xi(t)\right. \\
& \left.-C_{1} \int_{t-\tau(t)}^{t} \dot{\xi}(s) d s\right]^{\mathrm{T}} \times R\left[\left(C_{0}+C_{1}\right) \xi(t)\right. \\
& \left.-C_{1} \int_{t-\tau(t)}^{t} \dot{\xi}(s) d s\right]=\xi^{\mathrm{T}}(t)\left[\left(C_{0}+C_{1}\right)^{\mathrm{T}} G\right. \\
& +G\left(C_{0}+C_{1}\right)+E+\tau(t) G C_{1} R^{-1} C_{1}^{\mathrm{T}} G+G R^{-1} G \\
& +\tau(t)\left(C_{0}+C_{1}\right)^{\mathrm{T}} R\left(C_{0}+C_{1}\right)
\end{aligned}
$$

$$
\begin{aligned}
& \left.-2 \tau(t)\left(C_{0}+C_{1}\right)^{\mathrm{T}} R C_{1}+\tau(t) C_{1}{ }^{\mathrm{T}} R C_{1}\right] \xi(t)-(1 \\
& -h) \xi^{\mathrm{T}}(t-\tau(t)) E \xi(t-\tau(t))+\tau(t) \xi^{\mathrm{T}}(t-\tau(t)) \\
& \cdot C_{1}{ }^{\mathrm{T}} R C_{1} \xi(t-\tau(t))+2 \tau(t) \xi^{\mathrm{T}}(t)\left(C_{0}+C_{1}\right)^{\mathrm{T}} \\
& \cdot R C_{1} \xi(t-\tau(t))-\tau(t) \xi^{\mathrm{T}}(t) C_{1}^{\mathrm{T}} R C_{1} \xi(t-\tau(t)) \\
& -\tau(t) \xi^{\mathrm{T}}(t-\tau(t)) C_{1}^{\mathrm{T}} R C_{1} \xi(t)
\end{aligned}
$$

Letting $\Gamma(t)=\xi(t)-\xi(t-\tau(t))$, we have

$$
\dot{V}_{2}(t) \leq\left[\begin{array}{ll}
\xi^{\mathrm{T}}(t) & \Gamma^{\mathrm{T}}(t)
\end{array}\right]\left[\begin{array}{cc}
\Lambda_{11}+\tau^{*} G C_{1} R^{-1} C_{1}^{\mathrm{T}} G+G R^{-1} G & \Lambda_{12} \\
\Lambda_{21} & \Lambda_{22}
\end{array}\right]\left[\begin{array}{c}
\xi(t) \\
\Gamma(t)
\end{array}\right]
$$

According to inequality (37) and Lemma 2, it follows that

$$
\left[\begin{array}{cc}
\Lambda_{11}+\tau^{*} G C_{1} R^{-1} C_{1}^{\mathrm{T}} G+G R^{-1} G & \Lambda_{12} \\
\Lambda_{21} & \Lambda_{22}
\end{array}\right]<0
$$

Hence, $\dot{V}_{2}(t)<0$. According to Lyapunov theory, it can be concluded that the multiagent system (34) is asymptotically stable, that is

$$
\lim _{t \longrightarrow \infty} \xi(t)=0
$$

i.e., $\tilde{x}(t-\tau(t))=x_{i}(t-\tau(t))-x_{L}(t-\tau(t)) \longrightarrow 0$ and $\tilde{v}(t-\tau(t))=$ $v_{i}(t-\tau(t))-v_{L} \longrightarrow 0$, which demonstrates that the position and velocity of followers will asymptotically converge to that of the leader and thus the consensus is achieved even in the presence of time-varying delay.

\section{Simulation Study}

In this section, numerical simulations are carried out to verify the effectiveness of the proposed consensus algorithm with absent velocity measurement and time delay. Specifically, we choose one leader and four followers in the simulation.

The topological structure of multiagent system is illustrated in Figure 1, where only agent 1 and agent 2 are directly connected to the leader (labelled as agent 0 ). Then, we have

$$
\begin{aligned}
& \mathscr{L}=\left[\begin{array}{cccc}
2 & -1 & -1 & 0 \\
-1 & 2 & 0 & -1 \\
-1 & 0 & 2 & -1 \\
0 & -1 & -1 & 2
\end{array}\right], \\
& B=\left[\begin{array}{llll}
1 & 0 & 0 & 0 \\
0 & 1 & 0 & 0 \\
0 & 0 & 0 & 0 \\
0 & 0 & 0 & 0
\end{array}\right],
\end{aligned}
$$

$$
H=\left[\begin{array}{cccc}
3 & -1 & -1 & 0 \\
-1 & 3 & 0 & -1 \\
-1 & 0 & 2 & -1 \\
0 & -1 & -1 & 2
\end{array}\right]
$$

In the following, the consensus of second-order multiagent systems with constant time delay and time-varying delay is, respectively, performed to verify the correctness of the theoretical results.

5.1. Simulation Example 1: Constant Time Delay Case. In the constant time delay case, let $k_{1}=2, k_{2}=0.5$, and $k_{3}=0.3$. According to Theorem 8 , the maximum upper bound of time delay follows $\tau^{*}=0.002 \mathrm{~s}$ if $r=2$. The initial states of followers are $x(0)=[15,12,3,1] \mathrm{m}$ and $v(0)=[0,0,0,0] \mathrm{m} / \mathrm{s}$. The initial states of the leader are $x_{L}(0)=19 \mathrm{~m}$ and $v_{L}=$ $20 \mathrm{~m} / \mathrm{s}$, and the position of the leader updates according to (8). In addition, the feasible solution for the positive definite matrix $P$ can be solved by resorting to the LMI Toolbox in Matlab.

Based on the above simulation settings, we let $\tau=$ $0.001 \mathrm{~s}<\tau^{*}$. Under the consensus protocol (16), the simulation results are depicted in Figure 2.

From Figure 2, we can see that the proposed consensus protocol is able to achieve the consensus of second-order multiagent systems with absent velocity measurement and constant time delay. Under the consensus protocol (16) and the derived sufficient stability conditions (19) for constant time delay, the position errors of agents will asymptotically converge to zero. Meanwhile, their velocities will converge to that of the leader $(20 \mathrm{~m} / \mathrm{s})$, even though the neighbor's velocity is unknown to the followers.

5.2. Simulation Example 2: Time-Varying Delay Case. In the time-varying delay case, let $k_{1}=1, k_{2}=1, k_{3}=3$, and $\tau(t)=$ $0.35|\sin (t)|$. Hence, $\tau^{*}=0.35 \mathrm{~s}$ and $h=0.35$. The initial states of followers are $x(0)=[50,30,10,0] \mathrm{m}$ and $v(0)=$ 


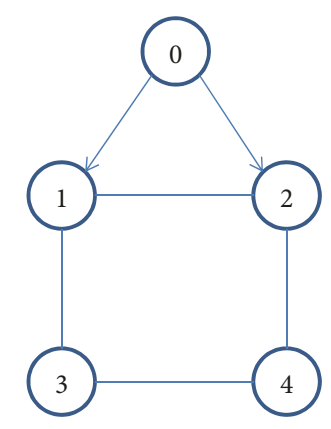

FIGURE 1: The topological structure of multiagent system.

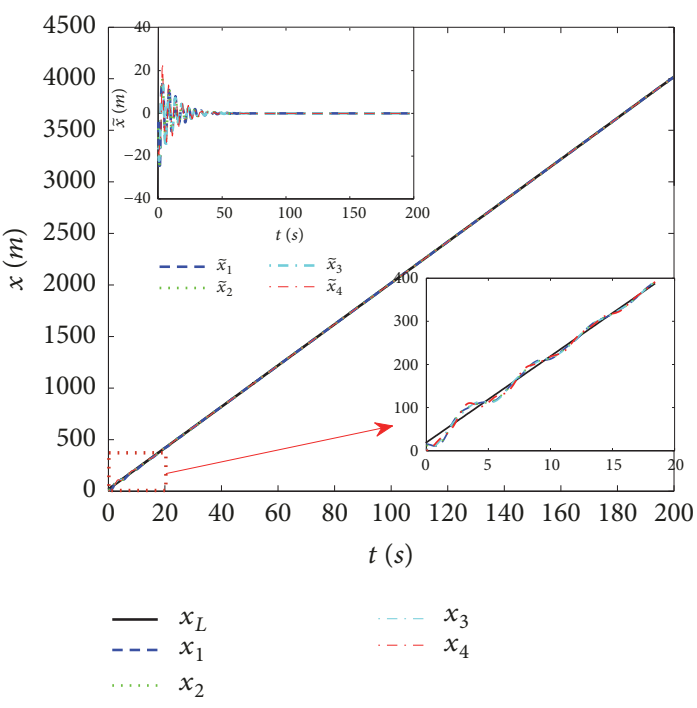

(a) Position

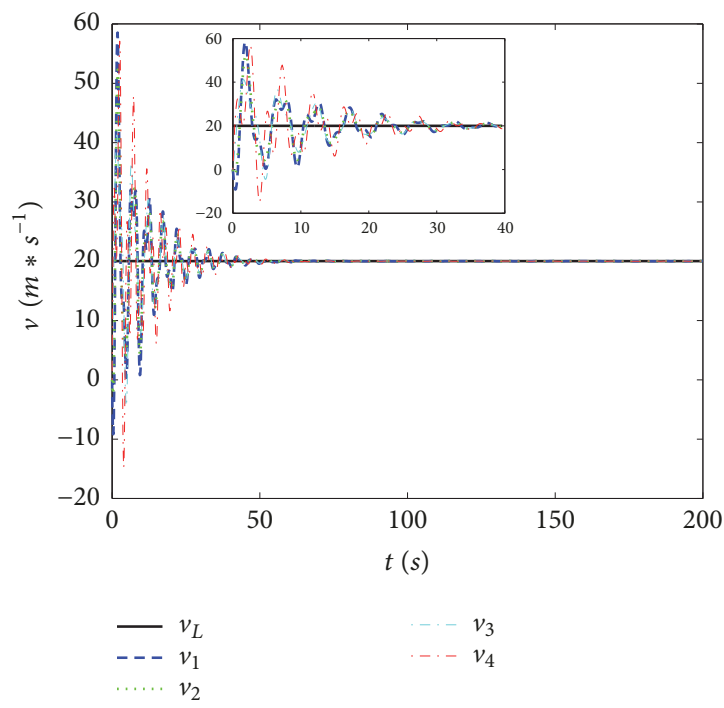

(b) Velocity

Figure 2: Positions and velocities of the leader and followers when the time delay $\tau=0.001 \mathrm{~s}$.

$[0,0,0,0] \mathrm{m} / \mathrm{s}$. The initial states of the leader are $x_{L}(0)=70 \mathrm{~m}$ and $v_{L}=20 \mathrm{~m} / \mathrm{s}$, and the position of the leader updates with (8). According to Theorem 10, the feasible solutions for the positive definite matrix $G, R, E$ can also be obtained by solving LMIs.

Based on the above simulation settings, we let $\tau(t)=$ $0.35|\sin (t)|$. Under the consensus protocol (34), the simulation results are depicted in Figure 3.

It can be seen from Figure 3 that the positions of followers will asymptotically converge to that of the leader and all the position errors approach zero within a finite time. In addition, the velocities of followers will be the same as the leader as time goes by. Therefore, the proposed algorithm (34) and the sufficient stability conditions (37) can achieve the consensus of second-order multiagent systems with absent velocity measurement and time-varying delay.

5.3. Comparative Analysis. In order to illustrate the superiority of the proposed consensus algorithm (12), a comparative simulation is carried out between Theorem 8 and [23]. In [23], the velocity information is assumed to be absent and other parameters are the same as that of Simulation Example
1. Under the consensus algorithm of Ref [23], the simulation results are shown in Figure 4.

The position curves of agents in Figure 4(a) suggest that the followers are not able to track the position of the leader. In addition, the velocities of agents, depicted in Figure 4(b), fluctuate intensively and cannot converge to the desired value. Therefore, compared with the consensus algorithm (16) proposed in this paper, the control law in [23] cannot realize the consensus when there is no velocity information measurement in the coordinated control of second-order multiagent systems.

The main reason lies in that the position information can only guarantee the position consensus of multiagent systems, whereas the velocity consensus cannot be ensured. By using the auxiliary system (11) proposed in this paper, the unavailable velocity information can be estimated and hence the velocity consensus can be achieved.

5.4. Further Discussion. In the above simulations, the underlying topology among followers is assumed to be undirected, which easily obtains a symmetric Laplacian matrix and hence facilitates the subsequent theoretical analysis. However, the 


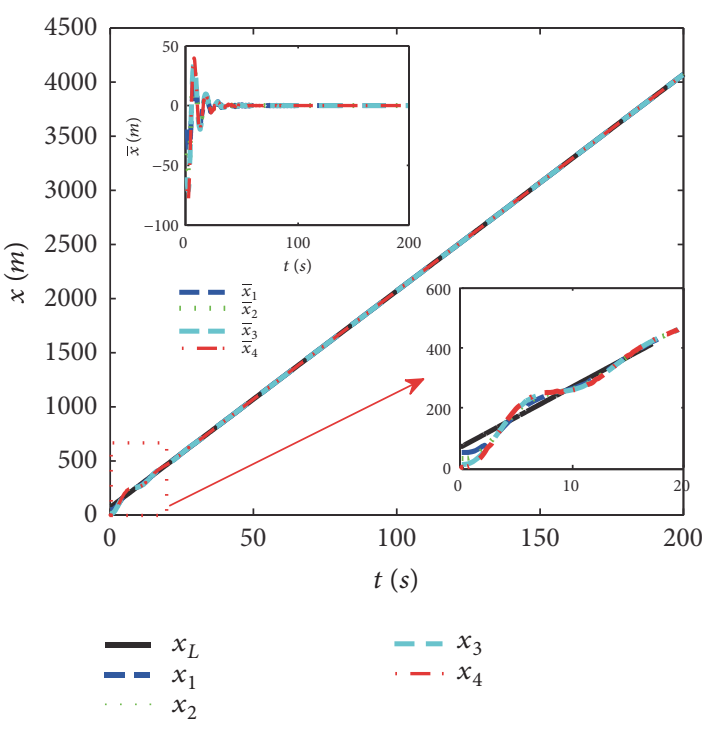

(a) Position

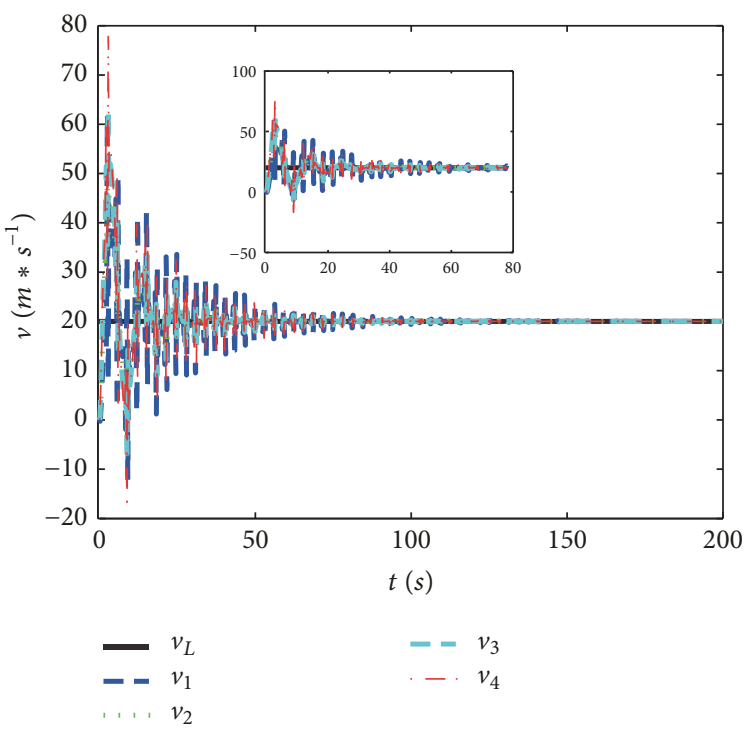

(b) Velocity

FIGURE 3: Positions and velocities of the leader and followers when the time delay $\tau(t)=0.35|\sin (t)| \mathrm{s}$.

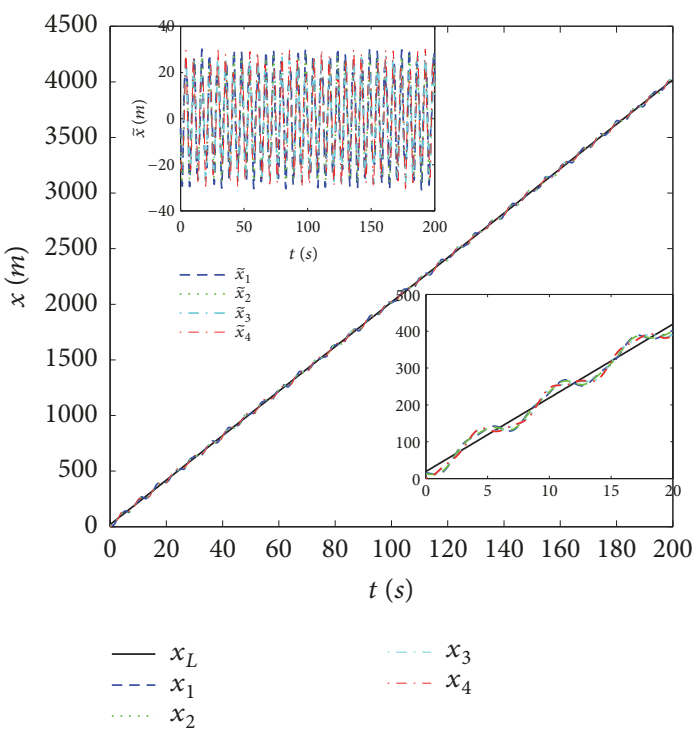

(a) Position

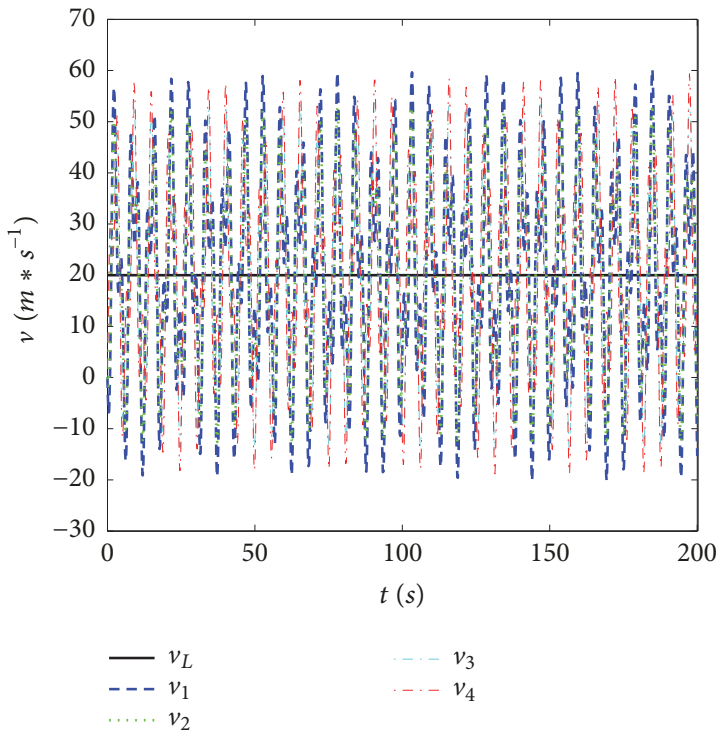

(b) Velocity

FIgURE 4: Positions and velocities of the leader and followers when velocity is unavailable in [23].

communication network between agents may be directed as in some cases agents can only receive information from others but not be able to send information to them. Therefore, it is of more significance to investigate the consensus problem of multiagent systems under directed topology.

In [29], the consensus of multiagent systems in directed networks with nonuniform time-varying delays is investigated with a tree-type transformation method. In [30], the second-order consensus problem for multiagent systems with nonlinear dynamics and directed topologies is discussed, where a new concept about the generalized algebraic connectivity is defined to describe the ability of reaching consensus in a directed network. Moreover, the global $\mathscr{H}_{\infty}$ pinning synchronization for a class of directed networks with aperiodic sampled-data communications is addressed in [31].

In general, the consensus of multiagent systems in directed networks requires that the communication graph of agents systems contains a spanning tree, which is more challenging than undirected topology.

\section{Conclusions and Future Work}

This paper investigates the leader-follower consensus problem of second-order multiagent systems, where the absent 
velocity measurement and time delay are considered simultaneously. For the absence of velocity information, an auxiliary system is designed to compensate for the unavailability of velocity measurement. Then, two time delay cases, including constant time delay and time-varying delay, are respectively, investigated and the sufficient conditions for the stability of the consensus algorithm are derived via LyapunovRazumkhin theorem and Lyapunov-Krasoviskii theorem. Simulation results show that the proposed consensus protocol is able to realize the consensus of multiagent systems even in the presence of absent velocity measurement and time delay.

It should be emphasized that the maximum allowable upper bound of time delay is a little conservative as the LMI approach adopted in this paper only derives the sufficient conditions for the stability of second-order multiagent system with time delay. Therefore, how to reduce conservatism on the estimation of the maximum allowable upper bound of time delay is our new concern in the future work.

\section{Data Availability}

The data used to support the findings of this study are available from the corresponding author upon request.

\section{Conflicts of Interest}

The authors declare that there are no conflicts of interest regarding the publication of this paper.

\section{Acknowledgments}

This work was partially supported by the National Natural Science Foundation of China (61803040), the Key Science and Technology Program of Shaanxi Province (2017JQ6060, 2018JQ6098), and the Fundamental Research Funds for the Central Universities of China (300102328403, 310832171004).

\section{References}

[1] T. Vicsek and A. Zafeiris, "Collective motion," Physics Reports, vol. 517, no. 3, pp. 71-140, 2012.

[2] W. Ren, "On consensus algorithms for double-integrator dynamics," IEEE Transactions on Automatic Control, vol. 53, no. 6, pp. 1503-1509, 2008.

[3] T. Vicsek, A. Czirk, E. Ben-Jacob, I. Cohen, and O. Shochet, "Novel type of phase transition in a system of self-driven particles," Physical Review Letters, vol. 75, no. 6, pp. 1226-1229, 1995.

[4] A. Jadbabaie, J. Lin, and A. S. Morse, "Coordination of groups of mobile autonomous agents using nearest neighbor rules," IEEE Transactions on Automatic Control, vol. 48, no. 6, pp. 988-1001, 2003.

[5] I. D. Couzin, C. C. Ioannou, G. Demirel et al., "Uninformed individuals promote democratic consensus in animal groups," Science, vol. 334, no. 6062, pp. 1578-1580, 2011.

[6] H. M. La, W. Sheng, and J. Chen, "Cooperative and active sensing in mobile sensor networks for scalar field mapping," IEEE Transactions on Systems, Man \& Cybernetics Systems, vol. 45, no. 1, pp. 1-12, 2014.
[7] X. Dong, B. Yu, Z. Shi, and Y. Zhong, "Time-varying formation control for unmanned aerial vehicles: theories and applications," IEEE Transactions on Control Systems Technology, vol. 23, no. 1, pp. 340-348, 2015.

[8] H. Pei, S. Chen, and Q. Lai, "Multi-target consensus circle pursuit for multi-agent systems via a distributed multi-flocking method," International Journal of Systems Science, vol. 47, no. 16, pp. 3741-3748, 2016.

[9] M. D. Yan, X. Zhu, X. X. Zhang, and Y. H. Qu, "Consensus-based three-dimensional multi-uav formation control strategy with high precision," Frontiers of Information Technology \& Electronic Engineering, vol. 18, no. 7, pp. 968-977, 2017.

[10] S. Caska and A. Gayretli, "An algorithm for collaborative patrolling systems with unmanned air vehicles and unmanned ground vehicles," in Proceedings of the 7th International Conference on Recent Advances in Space Technologies, RAST 2015, pp. 659-663, Istanbul, Turkey, June 2015.

[11] M. Yan, Y. Tang, P. Yang, and L. Zuo, "Consensus based platoon algorithm for velocity-measurement-absent vehicles with actuator saturation," Journal of Advanced Transportation, vol. 2017, Article ID 8023018, 8 pages, 2017.

[12] H. Su, X. Wang, and Z. Lin, "Flocking of multi-agents with a virtual leader," IEEE Transactions on Automatic Control, vol. 54, no. 2, pp. 293-307, 2009.

[13] A. S. Isira, Z. Zuo, and Z. Ding, "Leader-follower consensus control of Lipschitz nonlinear systems by output feedback," International Journal of Systems Science, vol. 47, no. 16, pp. 37723781, 2016.

[14] D. Zhao, T. Zou, S. Li, and Q. Zhu, "Adaptive backstepping sliding mode control for leader-follower multi-agent systems," IET Control Theory \& Applications, vol. 6, no. 8, pp. 1109-1117, 2012.

[15] W. Cao, J. Zhang, and W. Ren, "Leader-follower consensus of linear multi-agent systems with unknown external disturbances," Systems \& Control Letters, vol. 82, pp. 64-70, 2015.

[16] J. Mei, W. Ren, and G. Ma, "Distributed coordination for second-order multi-agent systems with nonlinear dynamics using only relative position measurements," Automatica, vol. 49, no. 5, pp. 1419-1427, 2013.

[17] H.-S. Su, "Flocking in multi-agent systems with multiple virtual leaders based only on position measurements," Communications in Theoretical Physics, vol. 57, no. 5, pp. 801-807, 2012.

[18] Y. Zheng and L. Wang, "Consensus of heterogeneous multiagent systems without velocity measurements," International Journal of Control, vol. 85, no. 7, pp. 906-914, 2012.

[19] X. Chen, H. Xu, and Y. Ban, "Rotating consensus of multi-agent systems without relative velocity measurement," Chinese Physics B, vol. 20, no. 9, Article ID 090515, pp. 148-151, 2011.

[20] P. Yang, M. Liu, X. Lei, and C. Song, "A novel control algorithm for the self-organized fission behavior of flocking system with time delay," International Journal of Control, Automation, and Systems, vol. 14, no. 4, pp. 986-997, 2016.

[21] P. Lin, M. Dai, and Y. Song, "Consensus stability of a class of second-order multi-agent systems with nonuniform timedelays," Journal of The Franklin Institute, vol. 351, no. 3, pp. 15711576, 2014.

[22] W. Yang, X. Wang, and H. Shi, "Fast consensus seeking in multiagent systems with time delay," Systems \& Control Letters, vol. 62 , no. 3, pp. 269-276, 2013.

[23] J. Hu and Y. Hong, "Leader-following coordination of multiagent systems with coupling time delays," Physica A: Statistical Mechanics and its Applications, vol. 374, no. 2, pp. 853-863, 2007. 
[24] W. Zhu and D. Cheng, "Leader-following consensus of secondorder agents with multiple time-varying delays," Automatica, vol. 46, no. 12, pp. 1994-1999, 2010.

[25] A. Shariati and M. Tavakoli, "A descriptor approach to robust leader-following output consensus of uncertain multi-agent systems with delay," Institute of Electrical and Electronics Engineers Transactions on Automatic Control, vol. 62, no. 10, pp. 5310-5317, 2017.

[26] S. Boyd, L. El Ghaoui, E. Feron, and V. Balakrishnan, Linear Matrix Inequalities in System and Control Theory, SIAM, Philadelphia, Pa, USA, 1994.

[27] J. K. Hale and S. M. Verduyn Lunel, Introduction to FunctionalDifferential Equations, vol. 99, Springer Science \& Business Media, 2013.

[28] B. Zhou and A. V. Egorov, "Razumikhin and Krasovskii stability theorems for time-varying time-delay systems," Automatica, vol. 71, pp. 281-291, 2016.

[29] Y. G. Sun and L. Wang, "Consensus of multi-agent systems in directed networks with uniform time-varying delays," IEEE Transactions on Automatic Control, vol. 54, no. 7, pp. 1607-1613, 2009.

[30] W. W. Yu, G. R. Chen, M. Cao, and J. Kurths, "Second-order consensus for multiagent systems with directed topologies and nonlinear dynamics," IEEE Transactions on Systems, Man, and Cybernetics, Part B: Cybernetics, vol. 40, no. 3, pp. 881-891, 2010.

[31] G. Wen, W. Yu, M. Z. Q. Chen, X. Yu, and G. Chen, " $H_{\infty}$ pinning synchronization of directed networks with aperiodic sampled-data communications," IEEE Transactions on Circuits and Systems I: Regular Papers, vol. 61, no. 11, pp. 3245-3255, 2014. 


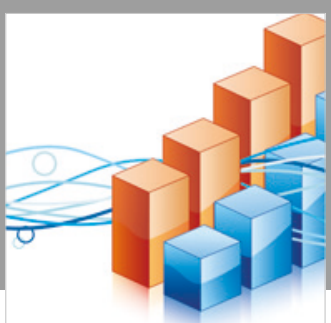

Advances in

Operations Research

\section{-n-m}
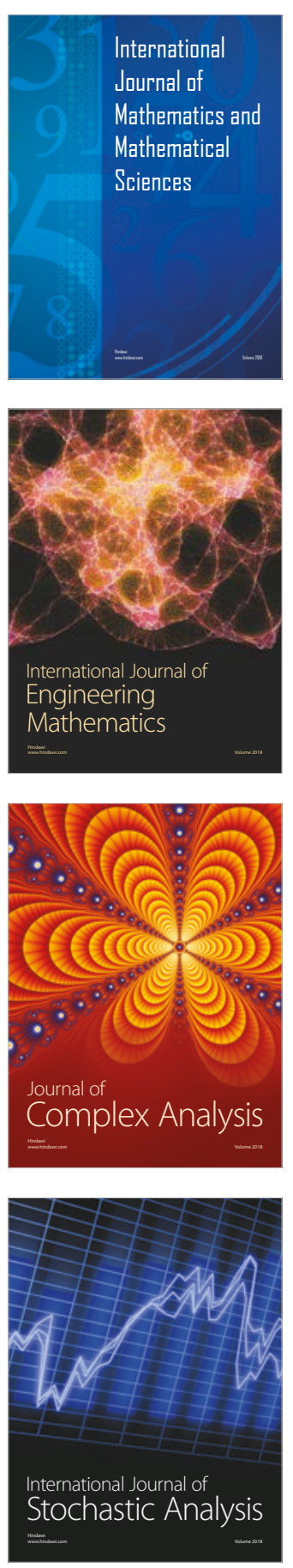
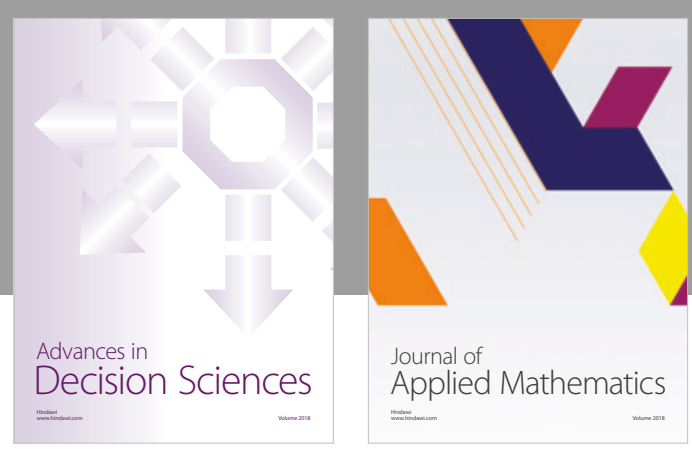

Journal of

Applied Mathematics
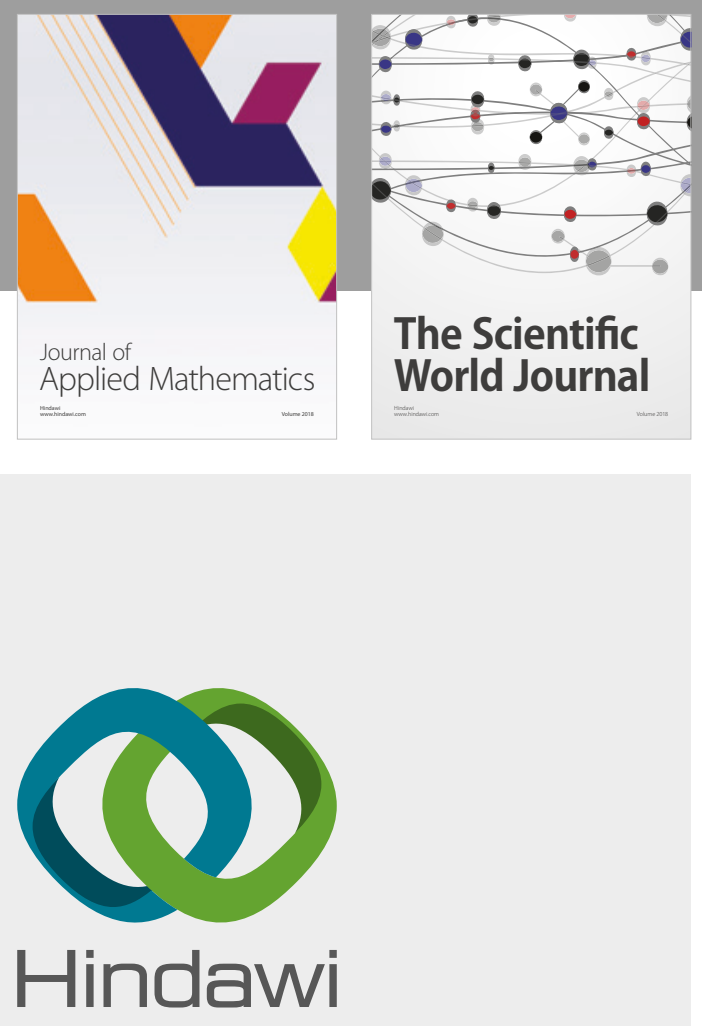

Submit your manuscripts at

www.hindawi.com

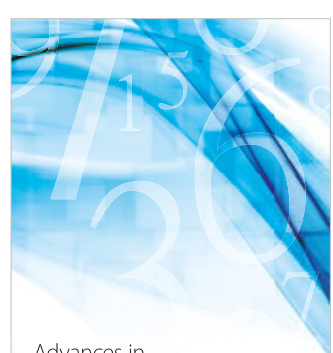

Advances in
Numerical Analysis
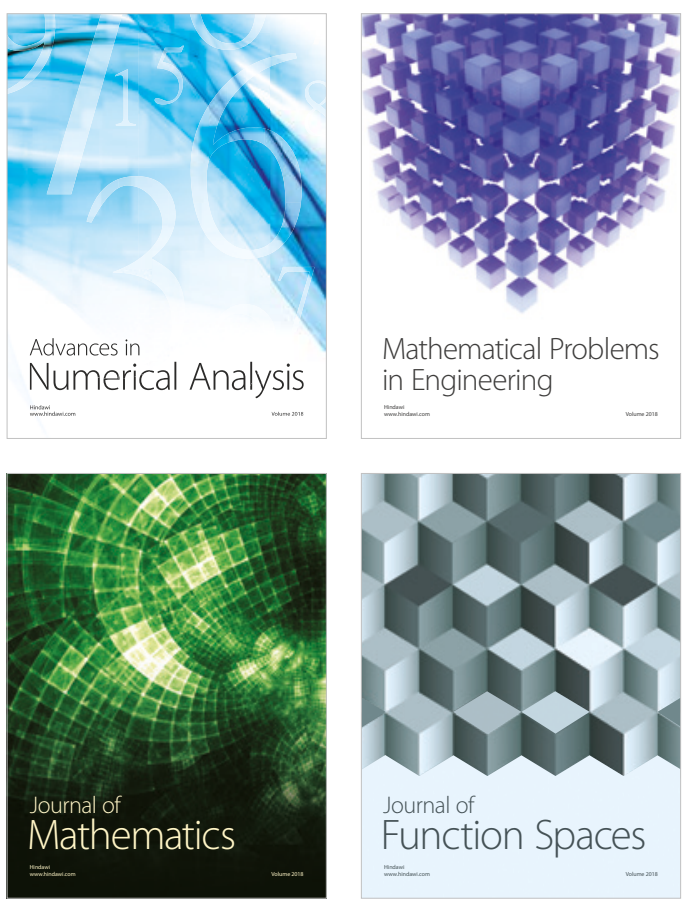

Mathematical Problems in Engineering

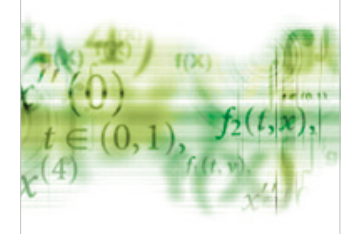

International Journal of

Differential Equations

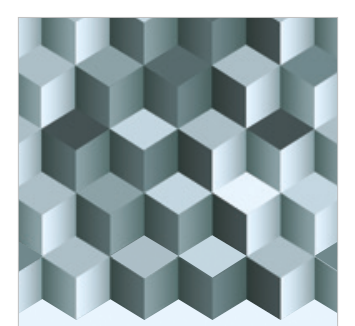

Journal of

Function Spaces

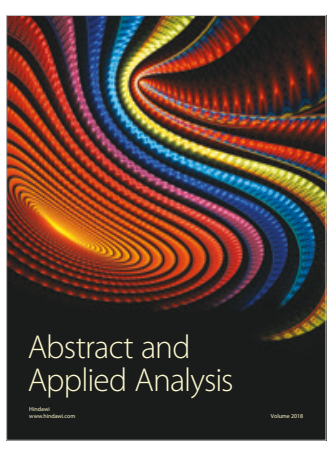

The Scientific

World Journal

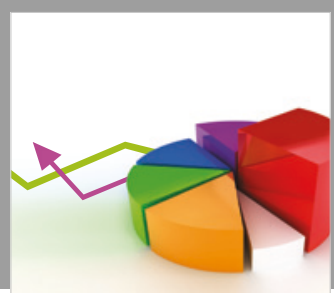

Journal of

Probability and Statistics
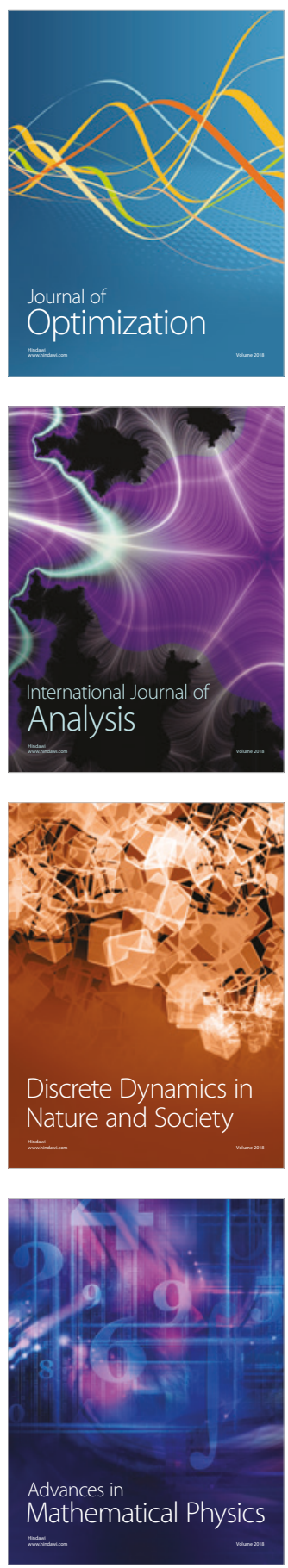